\title{
Nanofocusing of Surface Plasmons at the Apex of Metallic Tips and at the Sharp Metallic Wedges. Importance of Electric Field Singularity
}

\author{
Andrey Petrin \\ Joint Institute for High Temperatures of Russian Academy of Science \\ Russia
}

\section{Introduction}

Nanofocusing of light is localization of electromagnetic energy in regions with dimensions that are significantly smaller than the wavelength of visible light (of the order of one nanometer). This is one of the central problems of modern near-field optical microscopy that takes the resolution of optical imaging beyond the Raleigh's diffraction limit for common optical instruments [Zayats (2003), Pohl (1984), Novotny (1994), Bouhelier (2003), Keilmann (1999), Frey (2002), Stockman (2004), Kawata (2001), Naber (2002), Babadjanyan (2000), Nerkararyan (2006), Novotny (1995), Mehtani (2006), Anderson (2006)]. It is also important for the development of new optical sensors and delivery of strongly localized photons to tested molecules and atoms (for local spectroscopic measurements [Mehtani (2006), Anderson (2006), Kneipp (1997), Pettinger (2004), Ichimura (2004), Nie (1997), Hillenbrand (2002)]). Nanofocusing is also one of the major tools for efficient delivery of light energy into subwavelength waveguides, interconnectors, and nanooptical devices [Gramotnev (2005)].

There are two phenomena of exceptional importance which make it possible nanofocusing. The first is the phenomenon of propagation with small attenuation of electromagnetic energy of light along metal-vacuum or metal-dielectric boundaries. This propagation exists in the form of strictly localized electromagnetic wave which rapidly decreases in the directions perpendicular to the boundary. Remembering the quantum character of the surface wave they say about surface plasmons and surface plasmon polaritons (SPPs) as quasi-particles associated with the wave. The dispersion of the surface wave has the following important feature [Economou (1969), Barnes (2006)]: the wavelength tends to zero when the frequency of the SPPs tends to some critical (cut off) frequency above which the SPPs cannot propagate. For SPPs propagating along metal-vacuum plane boundary this critical frequency is equal to $\omega_{p} / \sqrt{2}$ (we use Drude model without absorption in metal). For spherical boundary this critical frequency [Bohren, Huffman (1983)] is equal to $\omega_{p} / \sqrt{3}$. So, the SPP critical frequency depends on the form of the boundary. By changing the frequency of SPPs it is possible to decrease the wavelength of the SPPs to the values substantially 
smaller than the wavelength of visible light in vacuum and use the SPPs for trivial focusing by creation a converging wave [Bezus (2010)]. In this case there is no breaking the diffraction Raleigh's limitation and the energy of the wave is focused into the region with dimensions of the order of wavelength of the SPPs. These dimensions may be substantially smaller than the wavelength of light in vacuum corresponding to the same frequency. As a result we have nanofocusing of light energy. The second phenomenon is electrostatic electric field strengthening at the apex of conducting tip (at the apex of geometrically ideal tip there is electrostatic field singularity, i.e. the electrostatic field tends to infinity at the apex). This phenomenon exists not only in electrostatics. For alternating electric field in the region with the apex of the tip at the center (with dimensions smaller than wavelength) the quasi-static approximation is applicable and there is a singularity of the time varying electric field (if the frequency is low enough as we will see below). Surely, at the apex of a real tip there is no singularity of electrostatic field since the apex is rounded. But near the apex the electric field increases in accordance with power (negative) law of the singularity and the electric field saturation at the apex is defined by the radius of the apex. This radius may be very small, of the order of atomic size.

Nanofocusing of SPPs at the apex of metal tip is considered in [Stockman (2004), De Angelis (2010)]. SPPs are created symmetrically at the basement of the tip and this surface wave converges along the surface of the metal to the tip's apex where surface wave energy is focused. But conditions for existence of electric field singularity are considered in [Stockman (2004), De Angelis (2010)] only for very sharp conical metal tips with small angle at the apex. In [Petrin (2010)] it is shown that due to frequency dependence of metal permittivity in optic frequency range the singularity of electric field at the tip's of not very sharp apex may exist in different forms.

The goal of the present chapter is investigation of the factors defined the type of singular concentration of electromagnetic energy at the geometrically singular metallic elements (such as apexes and edges) as one of the important condition for optimal nanofocusing.

In the next sections of this chapter we discuss the following:

electric field singularities in the vicinity of metallic tip's apex immersed into a uniform dielectric medium;

electric field singularities in the vicinity of metallic tip's apex touched a dielectric plate; electric field singularities in the vicinity of edge of metallic wedge.

\section{Nanofocusing of surface plasmons at the apex of metallic probe microtip. Conditions for electric field singularity at the apex of microtip immersed into a uniform dielectric medium.}

In this section of the chapter we focus our attention on finding the condition for electric field singularity of focused SPP electric field at the apex of a metal tip which is used as a probe in a uniform dielectric medium. As we have discussed above this singularity is an important feature of optimal SPP nanofocusing.

\subsection{Condition for electric field singularity at the apex}

Consider the cone surface of metal tip (see Fig. 1). 


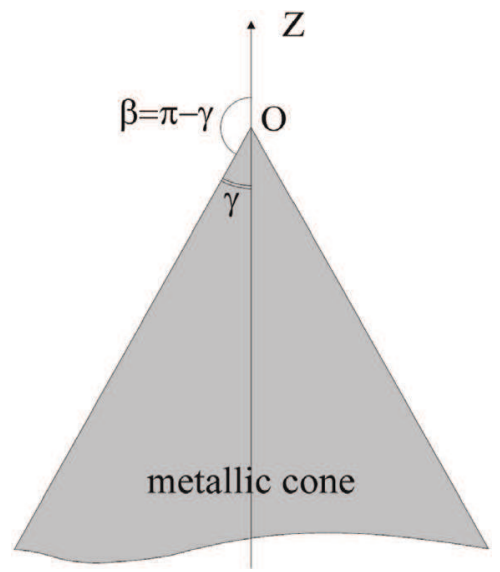

Fig. 1. Geometry of the problem.

Let calculate the electric field distribution near the tip's apex. In spherical coordinates with origin $O$ at the apex and polar angle $\theta$ (see Fig. 1), an axially symmetric potential $\Psi$ obeys Laplace's equation (we are looking for singular solutions, so in the vicinity of the apex the quasistatic approximation for electric field is applicable)

$$
\frac{\partial}{\partial r}\left(r^{2} \frac{\partial \Psi}{\partial r}\right)+\frac{1}{\sin \theta} \frac{\partial}{\partial \theta}\left(\sin \theta \frac{\partial \Psi}{\partial \theta}\right)=0
$$

Representing the solution as $\Psi=r^{\alpha} f(\theta)$, where $\alpha$ is a constant parameter, we have

$$
\frac{\partial}{\partial \theta}\left(\sin \theta \frac{\partial f}{\partial \theta}\right)=-\alpha(\alpha+1) f \sin \theta
$$

Changing to the function $g$ defined by the relation $f(\theta)=g(\cos \theta)$, which entails

$$
\frac{d f}{d \theta}=-\sin \theta \frac{d g}{d \cos \theta}
$$

we obtain Legendre's differential equation

$$
\left(1-\cos ^{2} \theta\right) \frac{d^{2} g}{d \cos ^{2} \theta}-2 \cos \theta \frac{d g}{d \cos \theta}+\alpha(\alpha+1) g=0 \text {. }
$$

It's solution is the Legendre polynomial $P_{\alpha}(\cos \theta)$ of degree $\alpha$, which can be conveniently represented as

$$
P_{\alpha}(\cos \theta)=F\left(-\alpha, \alpha+1,1, \frac{1-\cos \theta}{2}\right),
$$

where $F$ is the hypergeometric function. This representation is equally valid whether $\alpha$ is integer or not. 
Let denote the permittivity of metal cone as $\varepsilon_{m}$ and the permittivity of external uniform dielectric medium as $\varepsilon_{d}$. The electric potentials outside and inside the cone can be represented, respectively, as $\Psi_{d}=A r^{\alpha} P_{\alpha}(\cos \theta)$ and $\Psi_{m}=B r^{\alpha} P_{\alpha}(-\cos \theta)$, where $A$ and $B$ are constant parameters. Since the functions $P_{\alpha}(\cos \theta)$ and $P_{\alpha}(-\cos \theta)$ are linearly independent [Angot (1962)], the field components outside and inside the cone are, respectively,

$$
\begin{gathered}
E_{d, \tau}=-\frac{\partial \Psi_{d}}{\partial r}=-A \alpha r^{\alpha-1} P_{\alpha}(\cos \theta) \\
E_{d, n}=-\frac{1}{r} \frac{\partial \Psi_{d}}{\partial \theta}=A r^{\alpha-1} \sin \theta P_{\alpha}^{\prime}(\cos \theta)
\end{gathered}
$$

and

$$
\begin{gathered}
E_{m, \tau}=-\frac{\partial \Psi_{m}}{\partial r}=-B \alpha r^{\alpha-1} P_{\alpha}(-\cos \theta), \\
E_{m, n}=-\frac{1}{r} \frac{\partial \Psi_{m}}{\partial \theta}=-B r^{\alpha-1} \sin \theta P_{\alpha}^{\prime}(-\cos \theta) .
\end{gathered}
$$

At $\theta=\pi-\gamma$, the tangential and normal electric field components satisfy the boundary conditions

$$
E_{d, \tau}=E_{m, \tau}, \varepsilon_{d} E_{d, n}=\varepsilon_{m} E_{m, n}
$$

which yield the system of equations

$$
\begin{gathered}
A \alpha r^{\alpha-1} P_{\alpha}(\cos (\pi-\gamma))-B \alpha r^{\alpha-1} P_{\alpha}(-\cos (\pi-\gamma))=0, \\
\varepsilon_{d} A r^{\alpha-1} \sin (\pi-\gamma) P_{\alpha}^{\prime}(\cos (\pi-\gamma))+\varepsilon_{m} B r^{\alpha-1} \sin (\pi-\gamma) P_{\alpha}^{\prime}(-\cos (\pi-\gamma))=0,
\end{gathered}
$$

where the primes denote derivatives with respect to the arguments.

A nontrivial solution of the system exists when the determinant is equal to zero,

$$
\left(\varepsilon_{m} / \varepsilon_{d}\right)^{-1} P_{\alpha}(\cos \gamma) \frac{d}{d \gamma} P_{\alpha}(-\cos \gamma)-P_{\alpha}(\cos (\pi-\gamma)) \frac{d}{d \gamma} P_{\alpha}(\cos \gamma)=0
$$

Numerical calculation of the functions in Eq.(1) may be carried out with the aid of the hypergeometric function. Taking into account the following identity [Olver (1974)]

$$
\frac{\partial}{\partial z} F(a, b, c, z)=a b F(a+1, b+1, c+1, z)
$$

we find

$$
\frac{\partial}{\partial \theta} P_{\alpha}(\cos \theta)=-\alpha(\alpha+1) \frac{\sin \theta}{2} F\left(-\alpha+1, \alpha+2,2, \frac{1-\cos \theta}{2}\right)
$$




$$
\frac{\partial}{\partial \theta} P_{\alpha}(-\cos \theta)=\alpha(\alpha+1) \frac{\sin \theta}{2} F\left(-\alpha+1, \alpha+2,2, \frac{1+\cos \theta}{2}\right) .
$$

Therefore Eq.(1) may be rewritten as

$$
\begin{aligned}
\varepsilon_{m} F\left(-\alpha, \alpha+1,1, \frac{1+\cos \gamma}{2}\right) F\left(-\alpha+1, \alpha+2,2, \frac{1-\cos \gamma}{2}\right)+ \\
+\varepsilon_{d} F\left(-\alpha, \alpha+1,1, \frac{1-\cos \gamma}{2}\right) F\left(-\alpha+1, \alpha+2,2, \frac{1+\cos \gamma}{2}\right)=0 .
\end{aligned}
$$

For a given $\gamma$ Eq.(1) implicitly defines the function $\alpha=\alpha\left(\varepsilon_{m} / \varepsilon_{d}\right)$. Let use Drude model without losses for permittivity of metal $\varepsilon_{m}=1-\omega_{p}^{2} / \omega^{2}$ and consider that the metal tip surrounded with vacuum with permittivity $\varepsilon_{d}=1$, then $\varepsilon_{m} / \varepsilon_{d}=1-\omega_{p}^{2} / \omega^{2}$. In this case, $\alpha=\alpha\left(\omega / \omega_{p}\right)$ and remembering that $\Psi_{d} \sim r^{\alpha}$ we have $E_{d} \sim r^{\alpha-1}$. So, the function $\alpha=\alpha\left(\omega / \omega_{p}\right)$ defines the character of electric field singularity at the apex.

Note, that for given $\gamma$ and $\omega / \omega_{p}$ Eq.(1) has many roots but not all of them give solution which have physical sense or solution with electric field singularity at the apex of the tip. Obviously that only roots with $\operatorname{Re}(\alpha)<1\left(E_{d} \sim r^{\alpha-1}\right)$ will give singular solutions. So, we have interest only in these solutions. To find the lower boundary of the roots of interest it is necessary to remember that in the vicinity of a singular point the density of electric field power must be integrable value. It means that in the limit $r \rightarrow 0$ the electric field must increase slower than $r^{-3 / 2}$ and, therefore, the density of electric field power must increase slower than $r^{-3}$. It gives us the lower boundary for the roots. I.e. the appropriate roots must satisfy the inequity $\operatorname{Re}(\alpha)>-1 / 2$.

Eq.(1) was solved numerically. As an example of such calculations Fig. 2 shows real and image values of all roots of Eq.(1) in the interval $-1 / 2 \leq \operatorname{Re}(\alpha)<1$ as functions of $\omega / \omega_{p}$ for $\gamma=15^{\circ}, \varepsilon_{d}=1$ (solid curves). The dielectric constant of the metal was calculated by Drude formula without losses $\varepsilon_{m}=1-\omega_{p}^{2} / \omega^{2}$. From Fig. 2 we may see that there is a critical frequency of SPP $\omega_{c r}$ which separates the pure real solution (for $\omega<\omega_{c r}$ ) from essentially complex solution (for $\omega>\omega_{c r}$ ). It follows that there are two essentially different types of the SPP nanofocusing. First type takes place when $\omega<\omega_{c r}$ and is characterized by the electric field singularity without oscillations along radius in the vicinity of the apex. Second type takes place when $\omega_{c r}<\omega<\omega_{p} / \sqrt{2}$ and is characterized by the electric field singularity with oscillations along radius and the wave number of these oscillations tends to infinity when $r \rightarrow 0$. Note, that in the second type of nanofocusing $\operatorname{Re}(\alpha) \equiv-1 / 2$ if there is no losses in metal. The integral of the total electric power in the vicinity of the apex diverges. But if we take into account the metal losses considering the Drude's formula with losses

$$
\varepsilon_{m}=1-\frac{\omega_{p}^{2}}{\omega^{2}+i \Gamma \omega}, \text { where } \omega_{p} \text { and } \Gamma \text { are constants, }
$$

then in the second type of nanofocusing the integral of the total electric power in the vicinity of the apex converges since $\operatorname{Re}(\alpha)>-1 / 2$. As an example of the metal with losses was made silver $\left(\omega_{p}=1.36 \times 10^{16} \mathrm{~s}^{-1}\right.$ и $\Gamma=2 \times 10^{14} \mathrm{~s}^{-1}$ [Gay (2007)]). In Fig. 2 the curves for silver are shown by dotted lines. 
Note, that here there is an essential difference of the quasistatic approach to the problem from the pure static one. In the static problem physical sense have electric potentials with $\operatorname{Re}(\alpha)>0$ since only under this condition the potential on the surface of conducting cone may be made constant. In the quasistatic problem the equipotentiality of the metallic cone is not a necessary condition since the metal of the cone is considered as a dielectric with frequency dependent permittivity. The dielectric surface is not obligatory equipotential.

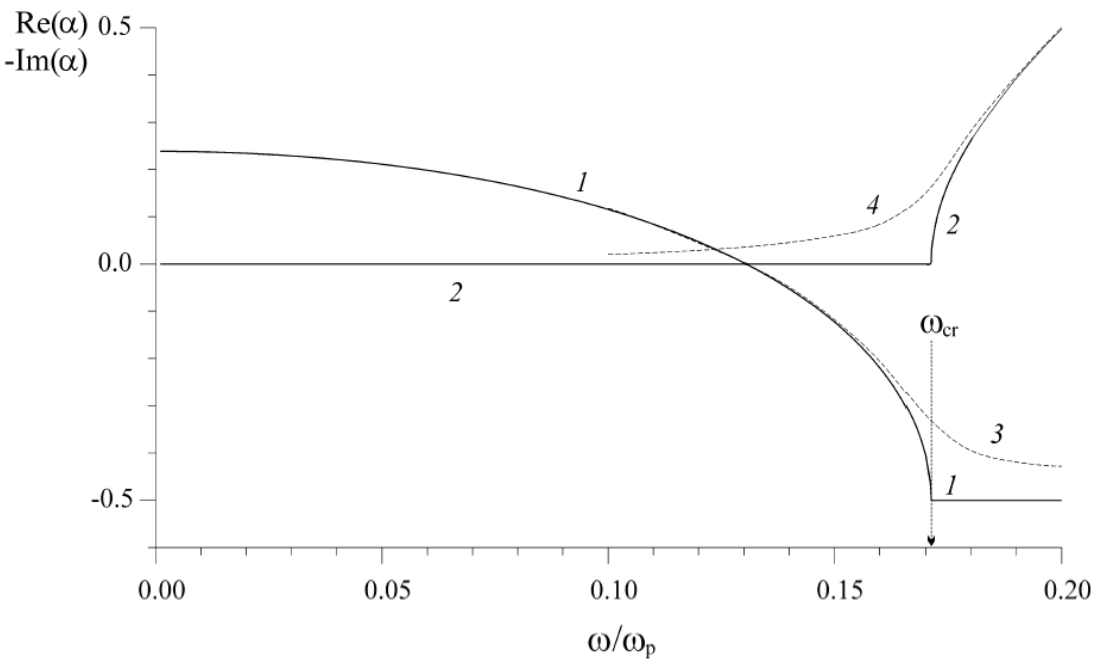

Fig. 2. Real (curve 1) and image (curve 2) parts of index $\alpha$ as a function of normalized frequency $\omega / \omega_{p}$ for of tip's angle $\gamma=15^{\circ}$ (no losses in metal). Dotted lines (curve 3 and 4 ) show analogues dependences for silver (metal with losses).

From the functions of Fig. 2, the normalized critical frequency $\omega_{c r}$ as a function of angle $\gamma$ was found numerically (see Fig.3). This function may be found analytically. If we take in Eq.(1) $\alpha=-1 / 2$ и $\varepsilon_{m} / \varepsilon_{d}=1-\omega_{p}^{2} / \omega_{c r}^{2}$ (the characteristics of critical points) then

$$
\omega_{c r} / \omega_{p}=\left[1+\frac{F\left(1 / 2,1 / 2,1, \frac{1-\cos \gamma}{2}\right) F\left(3 / 2,3 / 2,2, \frac{1+\cos \gamma}{2}\right)}{F\left(1 / 2,1 / 2,1, \frac{1+\cos \gamma}{2}\right) F\left(3 / 2,3 / 2,2, \frac{1-\cos \gamma}{2}\right)}\right]^{-\frac{1}{2}} .
$$

In Fig. 3 the frequency $\omega_{p} / \sqrt{2}$ is shown. SPPs can not exist above this frequency on the plane boundary metal-vacuum. We may see that when $\gamma \rightarrow 90^{\circ}$ the critical frequency tends to $\omega_{p} / \sqrt{2}$. It is absolutely unexpectable that the utmost frequency of SPP existence arises in quasistatic formulation of the problem on electric field singularity finding. 


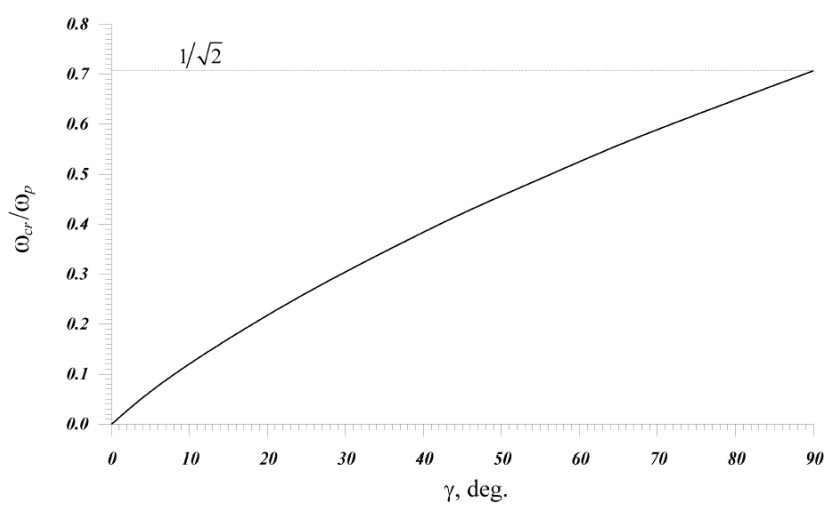

Fig. 3. Normalized critical frequency as a function of $\gamma$.

\subsection{Application of the theory to a silver tip.}

Consider a silver tip with plasma frequency [Fox (2003)] $\omega_{p}=1.36 \times 10^{16} \mathrm{~s}^{-1}$. Based on the function of Fig.3 we may find the function $\gamma_{c r}=\gamma_{c r}\left(\lambda_{0}\right)$ for silver by simple recalculating (see Fig. 4). The wavelength $\lambda_{0}$ is defined through the critical frequency $\omega_{c r}$ as $\lambda_{0}=2 \pi c / \omega_{\text {cr }}$, where $c$ is the speed of light in vacuum The curve plotted in Fig. 4 separates the region with the first type of singularity (above the curve) and the region with the second type of singularity (below the curve).

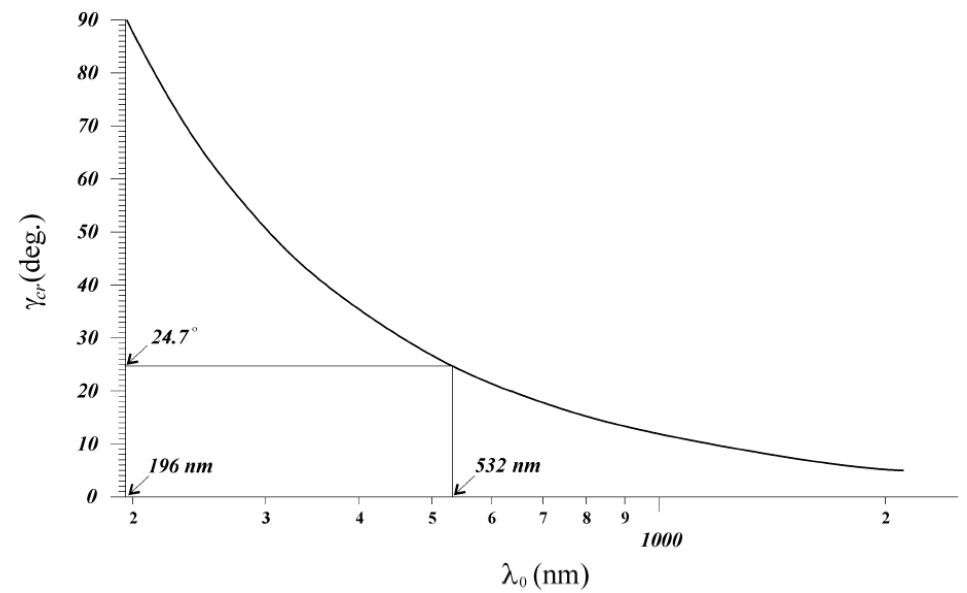

Fig. 4. Critical angle $\gamma_{c r}$ as a function of wavelength in vacuum of exciting laser. The curve starts from the lowest wavelength (in vacuum) of SPPs spectrum for silver: $\left(\lambda_{s p}\right)_{c r}=2 \sqrt{2} \pi c / \omega_{p} \approx 196 \mathrm{~nm}$.

As an example of application of the obtained results we consider the experiment on local Raman's microscopy [De Angelis (2010)]. A silver tip with angle $\gamma \approx 12^{\circ}$ in the vicinity of 
the apex was used. The wavelength of laser used in the experiment was equal to $\lambda_{0}=532 \mathrm{~nm}$. The excited SPPs propagated along the surface of the tip to the focus at the apex. But from the above results we may conclude that the angle is not optimal for the best focusing. Indeed, from Fig. 4 we may see that the critical angle for $\lambda_{0}=532 \mathrm{~nm}$ is $\gamma_{c r} \approx 24.7^{\circ}$. So, there is electric field singularity of the second type near the apex of the tip with $\gamma \approx 12^{\circ}$. So, it was obtained the fundamental result: the field singularity of alternative electric field at the apex of geometrically ideal metal cone tip exists in two form. First - with pure real index of singularity $\alpha$, and second - with essentially complex index of singularity with constant real part $\operatorname{Re}(\alpha)=-1 / 2$.

But it is remained the following unresolved problem yet. If the metal microtip is used as a SPP probe of the surface properties it is obvious that the value of the microtip's angle depends on the dielectric constant of the surface which the microtip's apex is touched and on the dielectric constant of the surrounding medium. It is clear that it is necessary to consider the influence of the probed surface on the electric field singularity at the microtip's apex and therefore on nanofocusing. The next section of this chapter gives the answer to the following question: what happens with the considered phenomenon when the apex of metal cone touches a dielectric plane plate?

\section{Nanofocusing of surface plasmons at the apex of metallic microtip probe touching a dielectric plane. Conditions for electric field singularity at the apex of a microtip immersed into uniform dielectric medium and touched a probed dielectric plane.}

In experiments the apex of the microtip may touch the surface of a probed dielectric plane plate (see Fig. 5). In this connection the following question arises: how the dielectric constant of the plate affects the electric field singularity index at the apex? By another words, if the microtip used as a concentrator of SPPs for Raman's spectroscopy of a dielectric surface [De Angelis (2010)] how the investigated material affects the electric field singularity at the apex and therefore the efficiency of SPPs focusing?

\subsection{Method of electric field singularity finding at the apex of metal microtip touching a dielectric plane}

Consider the cone metal microtip touching a dielectric plane plate (see Fig. 5). The space between the metal tip and dielectric plane is filled by a uniform dielectric. The dielectric constants of the metal, the dielectric plane and the filling uniform dielectric are equal to $\varepsilon_{m}$, $\varepsilon_{p}$ and $\varepsilon_{d}$ respectively. The frequency of SPP wave which is focused at the apex is equal to $\omega$. As in the previous part, in the quasistatic formulation of the problem the electric field potential must obeys Laplace's equation and normal and tangential components of electric field must obey the following boundary conditions: at the boundary of metal cone and free space $E_{m, \tau}=E_{d, \tau}, \varepsilon_{m} E_{m, n}=\varepsilon_{d} E_{d, n}$ and at the boundary of free space and dielectric plate $E_{d, \tau}=E_{p, \tau}, \varepsilon_{d} E_{d, n}=\varepsilon_{p} E_{p, n}$.

Based on the general solutions of Laplace's equation obtained in the previous part of this chapter, the electric potential in the considering three regions may be written as:

in the metal tip $\Psi_{m}=A r^{\alpha} P_{\alpha}(-\cos \theta)$; 


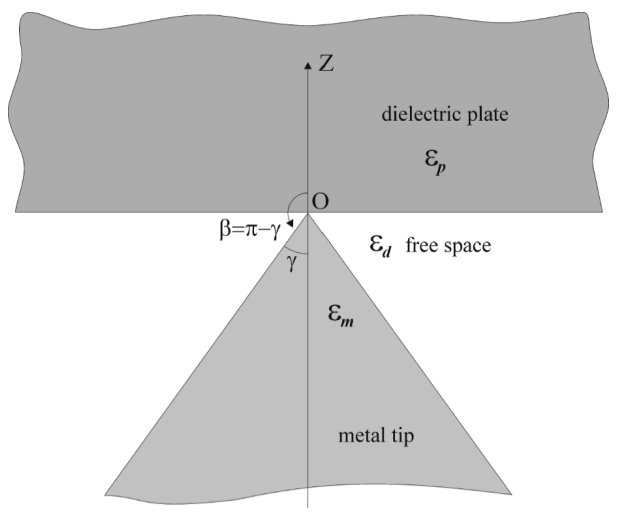

Fig. 5. Geometry of the problem.

in dielectric plate $\Psi_{p}=B r^{\alpha} P_{\alpha}(\cos \theta)$;

in free space $\Psi_{d}=C r^{\alpha} P_{\alpha}(\cos \theta)+D r^{\alpha} P_{\alpha}(-\cos \theta)$.

In these expressions we use the facts that for noninteger $\alpha$ the function $P_{\alpha}(\cos \theta)$ tends to infinity when $\theta \rightarrow \pi$ and $P_{\alpha}(-\cos \theta)$ tends to infinity when $\theta \rightarrow 0$.

Therefore, the field components in the considering three regions may be written by the following expressions:

tangential components:

$$
\begin{gathered}
E_{m, \tau}=-\frac{\partial \Psi_{m}}{\partial r}=-A \alpha r^{\alpha-1} P_{\alpha}(-\cos \theta), \\
E_{p, \tau}=-\frac{\partial \Psi_{p}}{\partial r}=-B \alpha r^{\alpha-1} P_{\alpha}(\cos \theta), \\
E_{d, \tau}=-\frac{\partial \Psi_{d}}{\partial r}=-C \alpha r^{\alpha-1} P_{\alpha}(\cos \theta)-D \alpha r^{\alpha-1} P_{\alpha}(-\cos \theta) ;
\end{gathered}
$$

normal components:

$$
\begin{gathered}
E_{m, n}=-\frac{1}{r} \frac{\partial \Psi_{m}}{\partial \theta}=-A r^{\alpha-1} \sin \theta P_{\alpha}^{\prime}(-\cos \theta), \\
E_{p, n}=-\frac{1}{r} \frac{\partial \Psi_{p}}{\partial \theta}=B r^{\alpha-1} \sin \theta P_{\alpha}^{\prime}(\cos \theta), \\
E_{d, n}=-\frac{1}{r} \frac{\partial \Psi_{d}}{\partial \theta}=C r^{\alpha-1} \sin \theta P_{\alpha}^{\prime}(\cos \theta)-D r^{\alpha-1} \sin \theta P_{\alpha}^{\prime}(-\cos \theta),
\end{gathered}
$$

where we use the natural notations:

$$
P_{\alpha}^{\prime}(\cos \theta)=d P_{\alpha}(\mu) /\left.d \mu\right|_{\mu=\cos \theta} \text { and } P_{\alpha}^{\prime}(-\cos \theta)=d P_{\alpha}(\mu) /\left.d \mu\right|_{\mu=-\cos \theta} .
$$


At the boundaries of the considering regions (at $\theta=\pi-\gamma$ and $\theta=\pi / 2$ ) the mentioned above boundary conditions for tangential and normal components of electric field must be satisfied. Substituting into the boundary conditions the expressions for the field components and taking into account that $-\cos (\pi-\gamma)=\cos \gamma$ we have the following four equations:

$$
\begin{gathered}
P_{\alpha}(\cos \gamma) A-P_{\alpha}(\cos (\pi-\gamma)) C-P_{\alpha}(\cos \gamma) D=0 \\
\varepsilon_{m} P_{\alpha}^{\prime}(\cos \gamma) A+\varepsilon_{d} P_{\alpha}^{\prime}(\cos (\pi-\gamma)) C-\varepsilon_{d} P_{\alpha}^{\prime}(\cos \gamma) D=0 \\
B-C-D=0 \\
-\varepsilon_{p} B+\varepsilon_{d} C-\varepsilon_{d} D=0
\end{gathered}
$$

where $P_{\alpha}^{\prime}(\cos (\pi-\gamma))=d P_{\alpha}(\mu) /\left.d \mu\right|_{\mu=\cos (\pi-\gamma)}$.

A nontrivial solution of the system exists when the determinant is equal to zero,

$$
\left|\begin{array}{cccc}
P_{\alpha}(\cos \gamma) & 0 & -P_{\alpha}(\cos (\pi-\gamma)) & -P_{\alpha}(\cos \gamma) \\
\left(\varepsilon_{m} / \varepsilon_{d}\right) P_{\alpha}^{\prime}(\cos \gamma) & 0 & P_{\alpha}^{\prime}(\cos (\pi-\gamma)) & -P_{\alpha}^{\prime}(\cos \gamma) \\
0 & 1 & -1 & -1 \\
0 & -\left(\varepsilon_{p} / \varepsilon_{d}\right) & 1 & -1
\end{array}\right|=0
$$

By expansion the determinant we have

$$
\begin{aligned}
& \left(\varepsilon_{m} / \varepsilon_{d}\right) P_{\alpha}^{\prime}(\cos \gamma)\left\{P_{\alpha}(\cos (\pi-\gamma))+P_{\alpha}(\cos \gamma)+\left(\varepsilon_{p} / \varepsilon_{d}\right)\left[P_{\alpha}(\cos (\pi-\gamma))-P_{\alpha}(\cos \gamma)\right]\right\}+ \\
& +P_{\alpha}(\cos \gamma)\left\{P_{\alpha}^{\prime}(\cos (\pi-\gamma))-P_{\alpha}^{\prime}(\cos \gamma)+\left(\varepsilon_{p} / \varepsilon_{d}\right)\left[P_{\alpha}^{\prime}(\cos (\pi-\gamma))+P_{\alpha}^{\prime}(\cos \gamma)\right]\right\}=0 .
\end{aligned}
$$

Numerical calculations of the functions in (2) were carried out with the aid of the hypergeometric function. Taking into account the identity [Olver (1974)]

$$
\frac{\partial}{\partial z} F(a, b, c, z)=a b F(a+1, b+1, c+1, z)
$$

we find

$$
\begin{aligned}
& \frac{\partial}{\partial \theta} P_{\alpha}(\cos \theta)=-\sin \theta d P_{\alpha}(\mu) /\left.d \mu\right|_{\mu=\cos \theta}=-\alpha(\alpha+1) \frac{\sin \theta}{2} F\left(-\alpha+1, \alpha+2,2, \frac{1-\cos \theta}{2}\right), \\
& \frac{\partial}{\partial \theta} P_{\alpha}(-\cos \theta)=\sin \theta d P_{\alpha}(\mu) /\left.d \mu\right|_{\mu=-\cos \theta}=\alpha(\alpha+1) \frac{\sin \theta}{2} F\left(-\alpha+1, \alpha+2,2, \frac{1+\cos \theta}{2}\right),
\end{aligned}
$$

and therefore

$$
d P_{\alpha}(\mu) /\left.d \mu\right|_{\mu=\cos \theta}=\frac{\alpha(\alpha+1)}{2} F\left(-\alpha+1, \alpha+2,2, \frac{1-\cos \theta}{2}\right),
$$




$$
d P_{\alpha}(\mu) /\left.d \mu\right|_{\mu=-\cos \theta}=\frac{\alpha(\alpha+1)}{2} F\left(-\alpha+1, \alpha+2,2, \frac{1+\cos \theta}{2}\right) .
$$

By substituting these expressions into (2) we have

$$
\begin{aligned}
& \frac{\varepsilon_{m}}{\varepsilon_{d}} F\left(-\alpha+1, \alpha+2,2, \frac{1-\cos \gamma}{2}\right) \times \\
& \quad\left\{\left(1+\frac{\varepsilon_{p}}{\varepsilon_{d}}\right) F\left(-\alpha, \alpha+1,1, \frac{1-\cos (\pi-\gamma)}{2}\right)+\left(1-\frac{\varepsilon_{p}}{\varepsilon_{d}}\right) F\left(-\alpha, \alpha+1,1, \frac{1-\cos \gamma}{2}\right)\right\}+ \\
& F\left(-\alpha, \alpha+1,1, \frac{1-\cos \gamma}{2}\right) \times \\
& \quad\left\{\left(1+\frac{\varepsilon_{p}}{\varepsilon_{d}}\right) F\left(-\alpha+1, \alpha+2,2, \frac{1-\cos (\pi-\gamma)}{2}\right)-\left(1-\frac{\varepsilon_{p}}{\varepsilon_{d}}\right) F\left(-\alpha+1, \alpha+2,2, \frac{1-\cos \gamma}{2}\right)\right\}=0 .
\end{aligned}
$$

If the particular case when $\varepsilon_{p} / \varepsilon_{d}=1$ is considered, this equation is transformed into

$$
\varepsilon_{d} P_{\alpha}(\cos \gamma) P_{\alpha}^{\prime}(-\cos \gamma)+\varepsilon_{m} P_{\alpha}(-\cos \gamma) P_{\alpha}^{\prime}(\cos \gamma)=0 .
$$

Since $P_{\alpha}^{\prime}(\cos \gamma)=-\sin ^{-1} \gamma \frac{d}{d \gamma} P_{\alpha}(\cos \gamma)$ and $P_{\alpha}^{\prime}(-\cos \gamma)=\sin ^{-1} \gamma \frac{d}{d \gamma} P_{\alpha}(-\cos \gamma)$ we may write

$$
\varepsilon_{d} P_{\alpha}(\cos \gamma) \frac{d}{d \gamma} P_{\alpha}(-\cos \gamma)-\varepsilon_{m} P_{\alpha}(\cos (\pi-\gamma)) \frac{d}{d \gamma} P_{\alpha}(\cos \gamma)=0 .
$$

Eq.(3) is identical to the corresponding Eq.(1) for the geometry without dielectric plate. In this case the cone tip with dielectric constant $\varepsilon_{m}$ is immersed into the uniform dielectric with constant $\varepsilon_{d}$. This problem has been solved for example in [Petrin (2007)].

The minimal root $\alpha$ of Eq.(2) (which corresponds to the physically correct solution) defines the character of electric field singularity in the vicinity of the cone apex. From Eq.(2) it follows that $\alpha$ is a function of three independent variables: the angle $\gamma$ and the ratios of dielectric constants $\varepsilon_{m} / \varepsilon_{d}$ and $\varepsilon_{p} / \varepsilon_{d}$, i.e. $\alpha=\alpha\left(\gamma, \varepsilon_{m} / \varepsilon_{d}, \varepsilon_{p} / \varepsilon_{d}\right)$. As it was shown below $\alpha=\alpha\left(\gamma, \varepsilon_{m} / \varepsilon_{d}, \varepsilon_{p} / \varepsilon_{d}\right)$ is a complex function even for real arguments (it is important).

Let use again Drude's model for permittivity of metal without absorption $\varepsilon_{m}=1-\omega_{p}^{2} / \omega^{2}$, where $\omega_{p}$ is the plasma frequency of the metal. Therefore, for fixed values of $\gamma, \varepsilon_{d}$ and $\varepsilon_{p}$, we may find the dependence $\alpha\left(\omega / \omega_{p}\right)$. Taking into account that $\Psi_{d} \sim r^{\alpha}$, we have $E_{e x} \sim r^{\alpha-1}$.

Note, that for fixed values of $\gamma, \varepsilon_{d}, \varepsilon_{p}$ and $\omega / \omega_{p}$ Eq.(1) has many roots $\alpha_{i}$ but not all of the roots have physical sense or represent the singular electric field at the cone apex. Obviously, that only roots smaller than unit $\left(E_{e x} \sim r^{\alpha-1}\right)$ give the singular electric field. So, we will be interested by the solutions of Eq.(2) in the interval $\operatorname{Re}(\alpha)<1$. To define the lower boundary of the solution's interval it is necessary to remind that in the vicinity of the apex the electric field density must be integrable. It means that the electric field and density must increase slower than $r^{-3 / 2}$ and $r^{-3}$ respectively when $r \rightarrow 0$. So, the lower boundary of the roots interval is equal to $1 / 2$ and the total roots interval of interest is $-1 / 2<\operatorname{Re}(\alpha)<1$. 
Eq.(2) was solved numerically. For $\gamma=15^{\circ}, \varepsilon_{d}=1$ and $\varepsilon_{p}=1$ the results of calculations are the same as in Fig. 2 obtained from Eq.(1). The plots for other values of dielectric constant of the plate $\varepsilon_{p}$ are analogous to the plot of Fig. 2.

Using the same approach as in the case of Fig. 3 it were calculated numerically (see Fig. 6) the dependences of the critical frequency $\omega_{c r}$ (normalized on the plasma frequency of the metal) on the cone angle $\gamma$ for $\varepsilon_{d}=1$ and several values of $\varepsilon_{p}$.

These dependences may be found analytically. The critical frequency $\omega_{c r}$ corresponds to the root value $\alpha=-1 / 2$. Substituting $\alpha=-1 / 2$ and $\varepsilon_{m}=1-\omega_{p}^{2} / \omega_{c r}^{2}$ into Eq.(2) we find the following expression which is valid for any values of $\varepsilon_{d}$ and $\varepsilon_{p}$ :

$$
\frac{\omega_{c r}}{\omega_{p}}=\left\{\begin{array}{c}
1+\varepsilon_{d} \frac{F\left(1 / 2,1 / 2,1, \frac{1-\cos \gamma}{2}\right)}{F\left(3 / 2,3 / 2,2, \frac{1-\cos \gamma}{2}\right)} \times \\
\quad \times \frac{\left(1+\frac{\varepsilon_{p}}{\varepsilon_{d}}\right) F\left(3 / 2,3 / 2,2, \frac{1+\cos \gamma}{2}\right)-\left(1-\frac{\varepsilon_{p}}{\varepsilon_{d}}\right) F\left(3 / 2,3 / 2,2, \frac{1-\cos \gamma}{2}\right)}{\left(1+\frac{\varepsilon_{p}}{\varepsilon_{d}}\right) F\left(1 / 2,1 / 2,1, \frac{1+\cos \gamma}{2}\right)+\left(1-\frac{\varepsilon_{p}}{\varepsilon_{d}}\right) F\left(1 / 2,1 / 2,1, \frac{1-\cos \gamma}{2}\right)}
\end{array}\right\}
$$

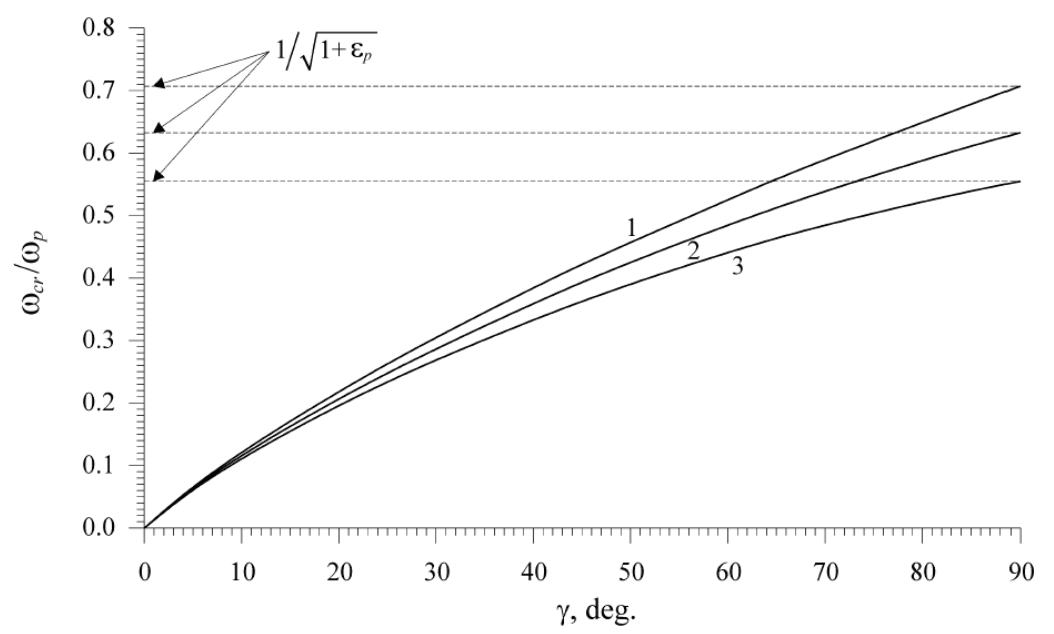

Fig. 6. Normalized critical frequency $\omega_{c r} / \omega_{p}$ as a function of $\gamma$ for $\varepsilon_{d}=1$ and several values of $\varepsilon_{p}$. Curve 1 for $\varepsilon_{p}=1,2-\varepsilon_{p}=1.5,3-\varepsilon_{p}=2.25$. It is shown the asymptotic of the curve when $\gamma \rightarrow 90^{\circ}$.

It was found that when $\gamma \rightarrow 90^{\circ}$ (the metal cone turns into metal plane and the free space between the cone and the dielectric plate disappears) the curves of the critical frequencies tends to the value $\omega_{p} / \sqrt{1+\varepsilon_{p}}$ - the utmost frequencies of SPP's existence on the boundary metal-dielectric plate [Stern (1960)]. As in the previous part of the chapter we see that it is 
absolutely unexpected that the utmost maximal frequency of SPP's existence arises in the quastatic statement of the singularity existence problem.

\subsection{Application of the theory to a silver tip}

So, as in the section 2.2, we see that if the working frequency is fixed, then there are two different types of singularity. In this case there is a critical angle $\gamma_{c r}$ which separates the regime with the first type of singularity from the regime with the second type of singularity. For $\varepsilon_{d}=1$ and several values of $\varepsilon_{p}$ the dependences $\gamma_{c r}=\gamma_{c r}\left(\lambda_{0}\right)$ of the critical angle on the wavelength of light in vacuum of the focused SPPs with frequency $\omega$ may be found from Fig. 6 by a recalculation as it was made (section 2.2) for microtip immersed into uniform medium. The plots $\gamma_{c r}=\gamma_{c r}\left(\lambda_{0}\right)$ for silver, $\varepsilon_{d}=1$ and for three values $\varepsilon_{p}=1, \varepsilon_{p}=1.5$ and $\varepsilon_{p}=2.25$ are shown in Fig. 7. Calculating the plots $\gamma_{c r}=\gamma_{c r}\left(\lambda_{0}\right)$ we neglect by losses in silver. If the angle of the cone $\gamma$ is more than $\gamma_{c r}$, then the singularity at the apex is of the first type. If the angle $\gamma$ is smaller than $\gamma_{c r}$, then the singularity is of the second type.

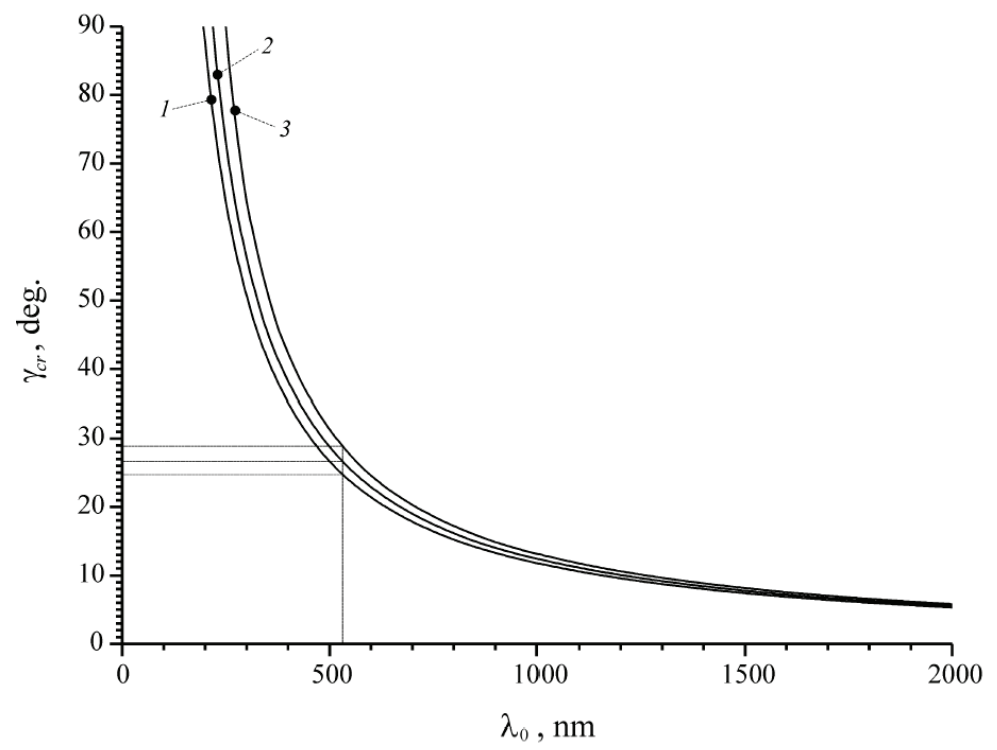

Fig. 7. Critical angle $\gamma_{c r}$ of silver cone as a function of wavelength in vacuum $\lambda_{0}$ of exciting laser for $\varepsilon_{d}=1$ and several values of $\varepsilon_{p}$. Curve 1 for $\varepsilon_{p}=1,2-\varepsilon_{p}=1.5,3-\varepsilon_{p}=2.25$. The left boundaries of the plots $\left(\lambda_{s p}\right)_{c r}=2 \pi c \sqrt{1+\varepsilon_{p}} / \omega_{p}$ are the limits of the spectrums of SPP on plane surface.

If (as in section 2.2) the wavelength of the laser $\lambda_{0}$, the dielectric constant of the working medium $\varepsilon_{d}$ and the dielectric constant of the dielectric plate $\varepsilon_{p}$ are given, then the cone angle $\gamma$ at the apex of focusing SPPs microtip defines the type of singularity. From Fig. 7 it may be seen that the more $\varepsilon_{p}$ the more $\gamma_{c r}$ under the other things being equal.

As in the previous section of the chapter, consider the setup of the work [De Angelis (2010)] on local Raman's microscopy. The waveength of the laser excited the focused SPPs is equal 
to $\lambda_{0}=532 \mathrm{~nm}$. The SPPs travel along the surface of the microtip cone and focus on its apex. From Fig. 7 it may be seen that the critical angle for $\varepsilon_{d}=1$ and $\varepsilon_{p}=1$ is equal to $\gamma_{c r} \approx 24.7^{\circ}$ (as in the previous section). If the dielectric constant $\varepsilon_{p}$ is equal to 1.5 or 2.25 then the critical angles are $\gamma_{c r} \approx 26.7^{\circ}$ and $\gamma_{c r} \approx 28.9^{\circ}$, respectively.

\section{Nanofocusing of surface plasmons at the edge of metallic wedge. Conditions for electric field singularity existence at the edge immersed into a uniform dielectric medium.}

In this part of the chapter we focus our attention on finding the condition for electric field singularity of focused SPP electric field at the edge of a metal wedge immersed into a uniform dielectric medium.

SPP nanofocusing at the apex of microtip (considered in the previous sections) corresponds (based on the analogy with conventional optics) to the focusing by spherical lens. Thus, SPP nanofocusing at the edge of microwedge corresponds to the focusing by cylindrical lens at the edge [Gramotnev (2007)]. The main advantage of the wedge SPP waveguide in nanoscale is the localization of plasmon wave energy in substantially smaller volume [Moreno (2008)] due to the electric field singularity at the edge of the microwedge. This advantage is fundamentally important for miniaturization of optical computing devices which have principally greater data processing rates in comparison with today state of the art electronic components [Ogawa (2008), Bozhevolnyi (2006)].

As it will be shown below the electric field singularity at the edge of the microwedge may be of two types due to frequency dependence of dielectric constant of metal in optical frequency range. This phenomenon is analogues to the same phenomenon for microtips which was considered in the previous parts of this chapter. The investigation of these types of electric field singularities at the edge of metal microwedge is the goal of this chapter section.

\subsection{Condition for electric field singularity at the edge of metallic wedge}

Let consider the metal microwedge (see Fig. 8) with dielectric constant of the metal $\varepsilon_{m}$. The frequency of the SPP is $\omega$. The wedge is immersed into a medium with dielectric costant $\varepsilon_{d}$.

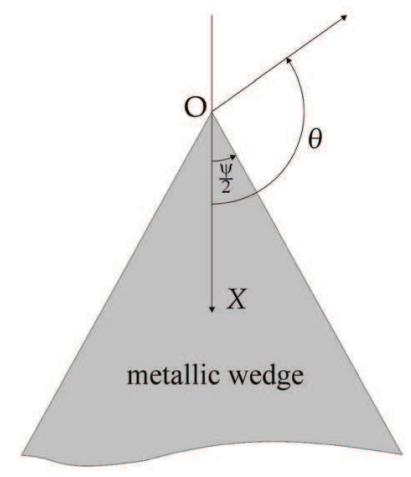

Fig. 8. Geometry of the wedge. 
Let calculate the electric field distribution near the edge. In cylindrical system of coordinates with origin $O$ and angle $\theta$ (see Fig. 8), a symmetric quasistatic potential $\varphi$ obeys Laplace's equation. The two independent solutions of the Laplace's equation are the functions $r^{\alpha} \sin (\alpha \theta)$ and $r^{\alpha} \cos (\alpha \theta)$ [Landau, Lifshitz (1982)] where $\alpha$ is a constant; $\theta$ is the angle from the axis $O X ; r$ is the radial coordinate from the origin $O$. Taking into account that the electric potential in the metal and dielectric depends on $r$ as the same power we may write the following expressions for potential in metal and dielectric respectively

$$
\begin{gathered}
\varphi=S_{m} r^{\alpha} \cos (\alpha \theta), \text { where }-\psi / 2 \leq \theta \leq \psi / 2, \\
\varphi=S_{d} r^{\alpha} \cos (\alpha(\theta-\pi)) \text {, where } \psi / 2 \leq \theta \leq(2 \pi-\psi / 2),
\end{gathered}
$$

where $S_{m}$ and $S_{d}$ are constants, $\psi$ is the total angle of the metallic wedge.

The boundary conditions for tangential and normal components of electric field may be written as

$$
E_{m, \tau}=E_{d, \tau} \text { and } \varepsilon_{m} E_{m, n}=\varepsilon_{d} E_{d, n} .
$$

Using the above expressions for electric potential in the two media the boundary conditions may be rewritten in the following form

$$
\begin{gathered}
E_{m, \tau}=-\frac{\partial \varphi}{\partial r}=-\alpha r^{\alpha-1} S_{m} \cos (\alpha \theta), \\
E_{d, \tau}=-\frac{\partial \varphi}{\partial r}=-\alpha r^{\alpha-1} S_{d} \cos (\alpha(\theta-\pi)), \\
E_{m, n}=-\frac{1}{r} \frac{\partial \varphi}{\partial \theta}=\alpha r^{\alpha-1} S_{m} \sin (\alpha \theta), \\
E_{d, n}=-\frac{1}{r} \frac{\partial \varphi}{\partial \theta}=\alpha r^{\alpha-1} S_{d} \sin (\alpha(\theta-\pi)) .
\end{gathered}
$$

At the first boundary of the wedge (where $\theta=\psi / 2$ ) the boundary conditions give two equations

$$
\begin{aligned}
& -\alpha r^{\alpha-1} S_{m} \cos (\alpha \theta)=-\left.\alpha r^{\alpha-1} S_{d} \cos (\alpha(\theta-\pi))\right|_{\theta=\psi / 2}, \\
& \varepsilon_{m} \alpha r^{\alpha-1} S_{m} \sin (\alpha \theta)=\left.\varepsilon_{d} \alpha r^{\alpha-1} S_{d} \sin (\alpha(\theta-\pi))\right|_{\theta=\psi / 2},
\end{aligned}
$$

or

$$
\begin{gathered}
S_{m} \cos (\alpha \psi / 2)=S_{d} \cos (\alpha(\psi / 2-\pi)), \\
\varepsilon_{m} S_{m} \sin (\alpha \psi / 2)=\varepsilon_{d} S_{d} \sin (\alpha(\psi / 2-\pi)) .
\end{gathered}
$$


Note, that at the second boundary of the wedge (where $\theta=-\psi / 2$ ) the boundary conditions give absolutely identical equations due to symmetry of the problem.

A nontrivial solution of the system exists when the determinant is equal to zero,

$$
\varepsilon_{m} \cos (\alpha(\psi / 2-\pi)) \sin (\alpha \psi / 2)-\varepsilon_{d} \sin (\alpha(\psi / 2-\pi)) \cos (\alpha \psi / 2)=0 \text {. }
$$

From this equation it follows that the index of singularity $\alpha$ is a function of two variables: angle $\psi$ and the ratio $\varepsilon_{m} / \varepsilon_{d}$, i.e. $\alpha=\alpha_{\min }\left(\psi, \varepsilon_{m} / \varepsilon_{d}\right)$.

Note, that in electrostatic field $\varepsilon_{m} \rightarrow \infty$ and, therefore, in the limit we have

$$
\cos (\alpha(\psi / 2-\pi)) \sin (\alpha \psi / 2)=0
$$

The minimal root of this equation will be when $\cos (\alpha(\psi / 2-\pi))=0$ (we are interested in the interval $\psi<\pi)$. Therefore, the minimal value of $\alpha$ is defined by equation $\alpha(\psi / 2-\pi)=-\pi / 2$ or $\alpha=\pi /(2 \pi-\psi)$ (it is well-known result [Landau, Lifshitz (1982)]). When $\psi \rightarrow 0$, we have $\alpha \rightarrow 1 / 2$.

Using Drude's model for permittivity of metal without absorption $\varepsilon_{m}=1-\omega_{p}^{2} / \omega^{2}$ and considering that the wedge is immersed into vacuum $\left(\varepsilon_{d}=1\right)$ we have $\varepsilon_{m} / \varepsilon_{d}=1-\omega_{p}^{2} / \omega^{2}$. Therefore, for fixed value of we may find the dependence $\alpha=\alpha_{\min }\left(\omega / \omega_{p}\right)$. Taking into account that $\varphi \sim r^{\alpha}$, we have $E_{e x} \sim r^{\alpha-1}$. Note, that from the physical sense of the electric potential $\varphi$ it follows that allways $\operatorname{Re}(\alpha) \geq 0$. Therefore, the interval $\alpha$ of singularity existence is $0 \leq \operatorname{Re}(\alpha)<1$.

Fig. 9 shows the dependences $\alpha=\alpha_{\min }\left(\omega / \omega_{p}\right)$ obtained from Eq.(4) for the wedge angle $\psi=30^{\circ}$. We can see that as in the case of cone tip in the case of wedge there are two types of electric field singularity at the edge of metallic wedges. The first type of electric field singularity takes place when $\omega<\omega_{c r}$. Here, the index $\alpha$ has a pure real value. The second type of electric field singularity takes place when $\omega>\omega_{c r}$ and the index $\alpha$ has a pure image value.

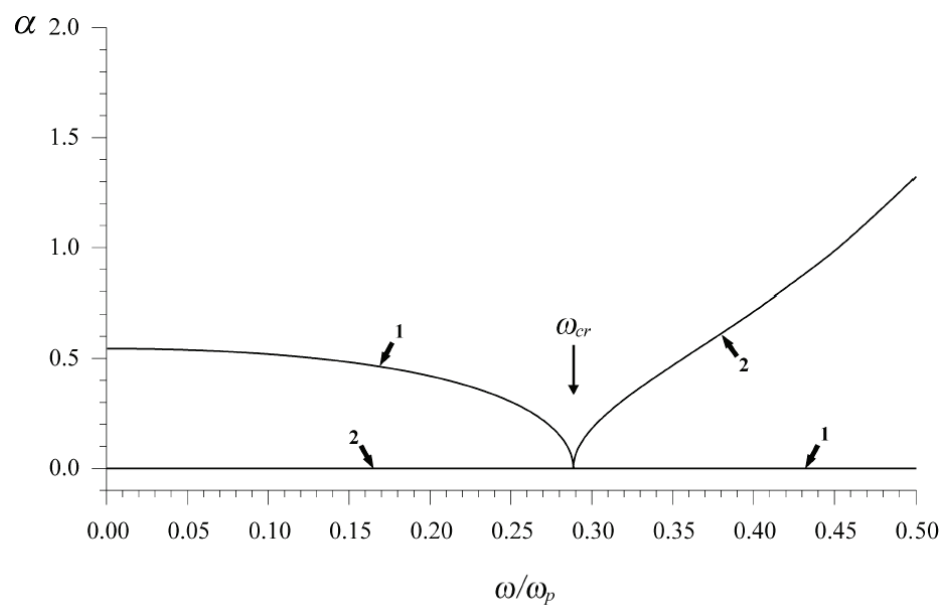

Fig. 9. Real (curve 1) and image (curve 2) part of the index $\alpha$ as a function of normalized frequency $\omega / \omega_{p}$ for the wedge angle $\psi=30^{\circ}$. 
Fig. 10 shows the plots of $\operatorname{Re}(\alpha)$ and $\operatorname{Im}(\alpha)$ as functions of $\omega / \omega_{p}$ (the same functions depicted in Fig.9) in the vicinity of the critical frequency $\omega_{c r}$ without losses. For comparison in Fig. 10 the plots of $\operatorname{Re}(\alpha)$ and $\operatorname{Im}(\alpha)$ as functions of $\omega / \omega_{p}$ for silver (the metal with losses) are shown.

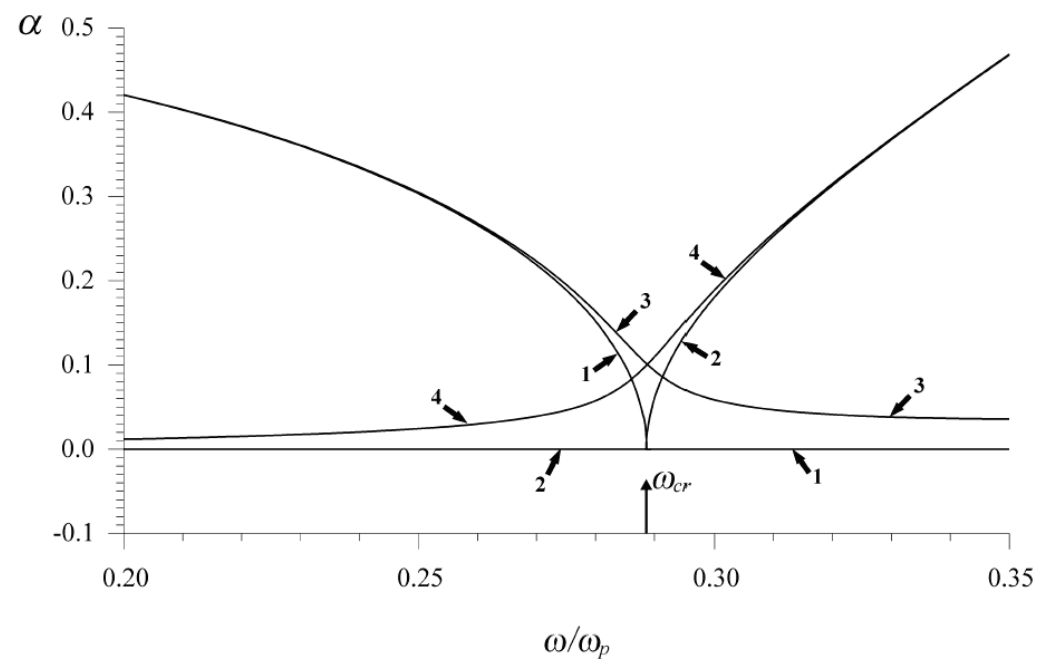

Fig. 10. The vicinity of the critical frequency $\omega_{c r}$. Real (curve 1) and image (curve 2) parts of the index $\alpha$ as a function of normalized frequency $\omega / \omega_{p}$ for the wedge angle $\psi=30^{\circ}$ and no losses in the metal. For comparison, the analogous curves 3 and 4 for silver wedge (metal with losses).

From the dependences like of Fig. 9 it was numerically found $\omega_{c r}$ (normalized on the plasma frequency $\omega_{p}$ ) as a function of the wedge angle $\psi$ (see Fig. 11). The obtained function is excellently approximated by the elementary function $\omega_{c r} / \omega_{p} \approx 0.05255 \sqrt{\psi[\mathrm{deg} .]}$. This is not a coincidence. Indeed, the condition $\omega=\omega_{c r}$ implies that $\alpha=0$ and therefore from Eq.(4) it follows

$$
\left(\varepsilon_{m}\right)_{c r}=\lim _{\alpha \rightarrow 0} \frac{\sin (\alpha(\psi / 2-\pi)) \cos (\alpha \psi / 2)}{\cos (\alpha(\psi / 2-\pi)) \sin (\alpha \psi / 2)}=1-\frac{2 \pi}{\psi} .
$$

Thus, $\omega_{c r}=\omega_{p} \sqrt{\psi / 2 \pi}$ or $\omega_{c r} / \omega_{p}=\sqrt{\psi[\mathrm{deg} .] / 360} \approx 0.0527046 \sqrt{\psi[\mathrm{deg} .]}$ exactly (analytical solution)! We can see that the accuracy of the numerical results is substantially high.

Fig. 11 also shows the frequency $\omega_{p} / \sqrt{2}$, above which SPP can not exist. Note, that when $\psi \rightarrow 180^{\circ}$ (the wedge turns into a plane) the critical frequency tends to $\omega_{p} / \sqrt{2}$ - the utmost frequency of SPP existence on plane surface. Again it is absolutely unexpectable that the utmost frequency of SPP existence arises in the quasistatic problem of singularity existence. 


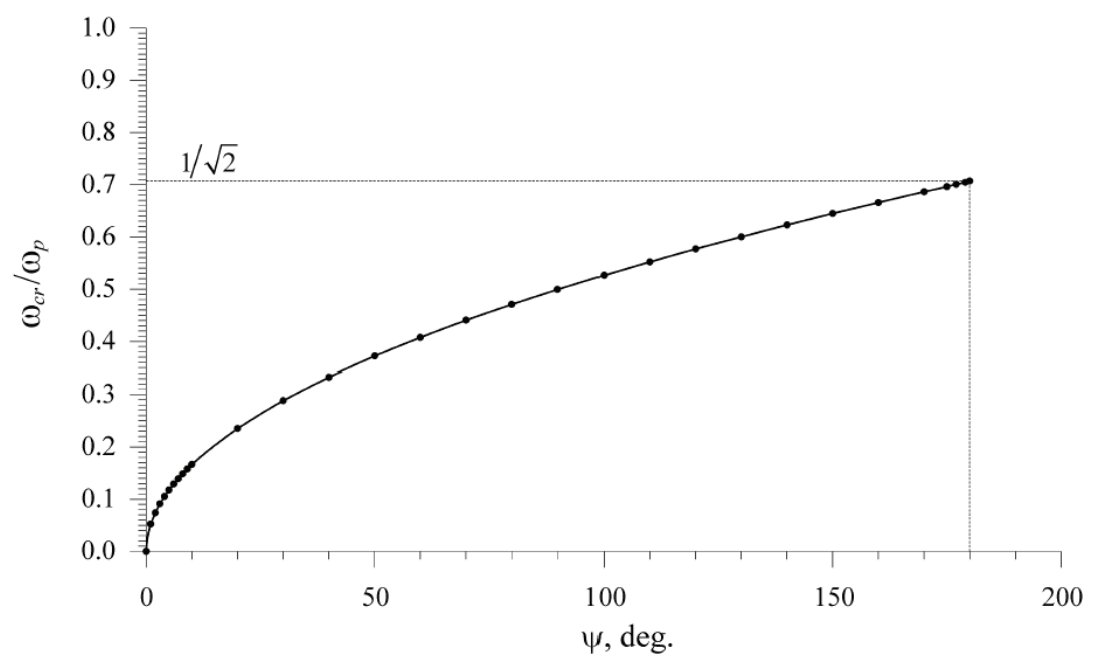

Fig. 11. Normalized critical frequency of singularity existence $\omega_{c r} / \omega_{p}$ as a function of the wedge angle $\psi$. Solid line is the approximating function $\omega_{c r} / \omega_{p} \approx 0.05255 \sqrt{\psi[\mathrm{deg} .]}$.

\subsection{Results of calculation for a silver microwegde}

From the results of the previous section 4.1 we see that the problem of finding of the SPP nanofocusing properties of microwedge is the following. On the one hand the wavelength of SPP must be possibly smaller. So, the SPP frequency must be close (but smaller) to the critical frequency of SPP existence $\omega_{p} / \sqrt{2}$. On the other hand it is necessary to use the effect of additional increasing of the SPP electric field at the edge of the microwedge due to electric field singularity at the edge. As we have seen the electric field singularity at the edge exists for any frequency of SPP, but there two different types of electric field singularity. The choice of the singularity depends on the particular technical problem (in this work this problems do not discuss).

Consider the following problem. Let there is a microwedge on the edge of which SPPs with frequency $\omega$ are focused (the wavelength in vacuum of a laser exciting the SPP is equal to $\left.\lambda_{0}\right)$. What is the value of wedge angle $\psi_{c r}$ which separates regimes of nanofocusing with different types of singularities?

Consider a microwedge made from silver (plasma frequency of silver is equal to $\omega_{p}=1.36 \times 10^{16} s^{-1}$ [Fox (2003)] and no losses). Based on the dependences of Fig. 11 it is elementary to find the function $\psi_{c r}=\psi_{c r}\left(\lambda_{0}\right)$ for silver (see Fig. 12) which is the solution of the considering problem. Indeed,

$$
\omega_{c r}=\omega_{p} \sqrt{\psi / 2 \pi} \Rightarrow 2 \pi c / \lambda_{0}=\omega_{p} \sqrt{\psi_{c r} / 2 \pi} \Rightarrow \psi_{c r}=\left(8 \pi^{3} c^{2} / \omega_{p}^{2}\right) / \lambda_{0}^{2}
$$

or for the angle in degrees

$$
\psi_{c r}[\operatorname{deg} .]=\left(1440 \pi^{2} c^{2} / \omega_{p}^{2}\right) / \lambda_{0}^{2} .
$$

Thus, we have obtained for this problem the exact analytical solution 


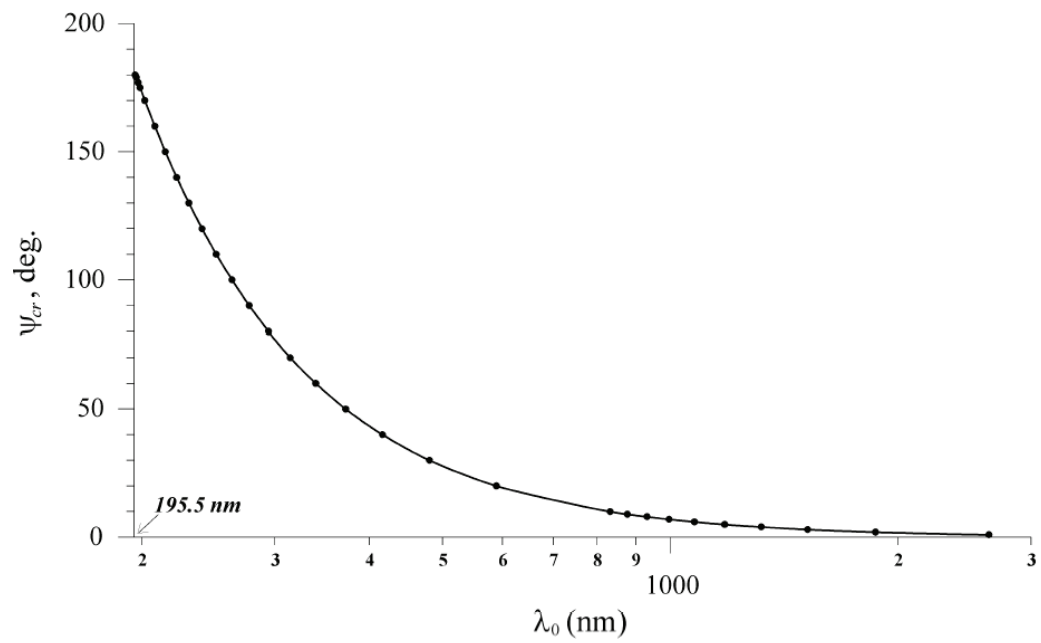

Fig. 12. Critical wedge angle $\psi_{c r}$ as a function of the SPP wavelength in vacuum. The left limit of the graphic $\left(\lambda_{s p}\right)_{c r}=2 \sqrt{2} \pi c / \omega_{p} \approx 195.5 \mathrm{~nm}$ - the utmost point of SPP spectrum for silver.

Thus, we may finish this section by the following fundamental statement: the field singularity of alternative electric field at the edge of geometrically ideal metal wedge exists in two forms. First - with pure real index of singularity $\alpha$, and second - with essentially image index of singularity with constant real part $\operatorname{Re}(\alpha)=0$. This phenomenon is due to the strong frequency dependence of metal permittivity at the range of light frequencies.

\section{Conclusion}

It was obtained the fundamental result: the electric field singularity of alternative electric field of SPP wave at the apex of geometrically ideal metal conical tip or at the edge of geometrically ideal metal wedge may exist in two forms depending on the type of the index of singularity. For a given SPP frequency and surrounding media the type the singularity defines by the angle of the tip (or wedge). This remains true for the case when the apex of metal cone touches a dielectric plane plate.

\section{References}

Anderson N., Bouhelier A., Novotny L. (2006) J. Opt. A Vol. 8, p. S227.

Angot A. (1962), Complements de mathematiques a l'usage des ingenieurs de l'electrotechnique et des telecommunications, La Revue d'Optique, Paris, 2nd ed.

Babadjanyan A. J., Margaryan N. L., Nerkararyan K. V. (2000) J. Appl. Phys. Vol. 87, p. 8.

Barnes W. L. (2006) J. Opt. A: Pure Appl. Opt. Vol. 8, p. S87.

Bezus E. A., Doskolovich L. L., Kazanskiy N. L. et al. (2010) J. Opt. Vol. 12, p. 015001.

Bohren C. F., Huffman D. R. (1983), Absorption and Scattering of Light by Small Particles, John Wiley \& Sons, New York.

Bouhelier A., Renger J., Beversluis M. R., Novotny L. (2003) J. Microsc. Vol. 210, p. 220. 
Bozhevolnyi S. I., Volkov V. S., Devaux E., et al. (2006) Nature, Vol. 440, p. 508.

De Angelis F., Das G., Candeloro P., et al. (2010) Nature Nanotech. Vol. 5, p. 67.

Economou E. N. (1969) Phys. Rev. Vol. 182, p.539.

Fox M. (2003), Optical Properties of Solids, Oxford University Press Inc., New York.

Frey H. G., Keilmann F., Kriele A., Guckenberger R. (2002) Appl. Phys. Lett. Vol. 81, p. 5030.

Gay G., Alloschery O., Weiner J., et al. (2007) Phys. Rev. E, Vol. 75, p. 016612.

Gramotnev D. K. (2005) J. Appl. Phys. Vol. 98, p. 104302.

Gramotnev D. K., Vernon K. C. (2007) Appl. Phys. B. Vol. 86, p. 7.

Hillenbrand R., Taubner T., Kellmann F. (2002) Nature Vol. 418, p. 159.

Ichimura T., Hayazawa N., Hashimoto M., et al. (2004) Phys. Rev. Lett. Vol. 92, p. 220801.

Kawata S. (2001), Near-Field Optics and Surface Plasmon-Polaritons, Springer, Berlin.

Keilmann F. (1999) J. Microsc. Vol. 194, p.567.

Kneipp K., Wang Y., Kneipp H., et al. (1997) Phys. Rev. Lett. Vol. 78, p. 1667.

Mehtani D., Lee N., Hartschuh R. D., et al. (2006) J. Opt. A Vol. 8, p. S183.

Moreno E., Rodrigo S. G., Bozhevolnyi S. I., et al. (2008) Phys. Rev. Lett. Vol. 100, p. 023901.

Landau L. D., Lifshitz E. M. (1982), Electrodynamics of continuos media, Nauka, Moscow.

Naber A., Molenda D., Fischer U. C., et al. (2002) Phys. Rev. Lett. Vol. 89, p. 210801.

Nerkararyan K. V., Abrahamyan T., Janunts E., et al. (2006) Phys. Lett. A Vol. 350, p. 147.

Nie S. M., Emory S. R. (1997) Science Vol. 275, p. 1102.

Novotny L., Hafner C. (1994) Phys. Rev. E Vol. 50, p. 4094.

Novotny L., Pohl D. W., Hecht B. (1995) Ultramicroscopy Vol. 61, p. 1.

Petrin A. B. (2007), Journal of Experimental and Theoretical Physics, Vol. 105, №6, p. 1236.

Petrin A. B. (2010), J. Nanoelectron. Optoelectron., Vol. 5, № 1, p. 55.

Petrin A. B. (2010), High Temperature (in press).

Pettinger B., Ren B., Picardi G., et al. (2004) Phys. Rev. Lett. Vol. 92, p. 096101.

Pohl D. W., Denk W., Lanz M. (1984) Appl. Phys. Lett. Vol. 44, p.651.

Ogawa T., Pile D. F. P., Okamoto T., et al. (2008) J. Appl. Phys. Vol. 104, p. 033102.

Olver F. W. J. (1974), Asymptotics and Special Functions, L.: Academic Press, New York.

Stern E. A., Ferrell R. A. (1960) Phys. Rev. Vol. 120, p. 130.

Stockman M. I. (2004) Phys. Rev. Lett. Vol. 93, p. 137404.

Zayats A. V., Smolyaninov I. (2003) J. Opt. A. Vol. 5, p. S16. 


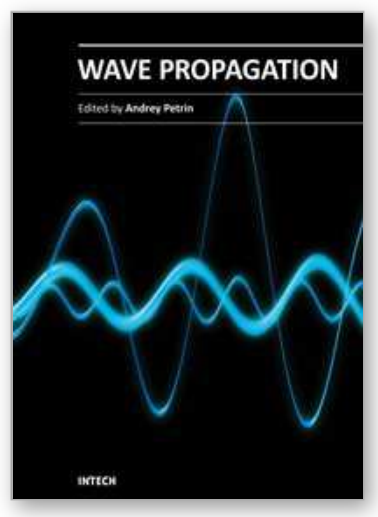

\author{
Wave Propagation \\ Edited by Dr. Andrey Petrin
}

ISBN 978-953-307-275-3

Hard cover, 570 pages

Publisher InTech

Published online 16, March, 2011

Published in print edition March, 2011

The book collects original and innovative research studies of the experienced and actively working scientists in the field of wave propagation which produced new methods in this area of research and obtained new and important results. Every chapter of this book is the result of the authors achieved in the particular field of research. The themes of the studies vary from investigation on modern applications such as metamaterials, photonic crystals and nanofocusing of light to the traditional engineering applications of electrodynamics such as antennas, waveguides and radar investigations.

\title{
How to reference
}

In order to correctly reference this scholarly work, feel free to copy and paste the following:

Andrey Petrin (2011). Nanofocusing of Surface Plasmons at the Apex of Metallic Tips and at the Sharp Metallic Wedges. Importance of Electric Field Singularity, Wave Propagation, Dr. Andrey Petrin (Ed.), ISBN: 978-953307-275-3, InTech, Available from: http://www.intechopen.com/books/wave-propagation/nanofocusing-ofsurface-plasmons-at-the-apex-of-metallic-tips-and-at-the-sharp-metallic-wedges-impor

\section{INTECH}

open science | open minds

\section{InTech Europe}

University Campus STeP Ri

Slavka Krautzeka 83/A

51000 Rijeka, Croatia

Phone: +385 (51) 770447

Fax: +385 (51) 686166

www.intechopen.com

\section{InTech China}

Unit 405, Office Block, Hotel Equatorial Shanghai

No.65, Yan An Road (West), Shanghai, 200040, China

中国上海市延安西路65号上海国际贵都大饭店办公楼405单元

Phone: +86-21-62489820

Fax: +86-21-62489821 
(C) 2011 The Author(s). Licensee IntechOpen. This chapter is distributed under the terms of the Creative Commons Attribution-NonCommercialShareAlike-3.0 License, which permits use, distribution and reproduction for non-commercial purposes, provided the original is properly cited and derivative works building on this content are distributed under the same license. 